\title{
Fuzzy Logic PID Control of a PMDCM Speed Connected to a 10-kW DC PV Array Microgrid-Case Study
}

\author{
Roxana-Elena Tudoroiu \\ University of Petrosani \\ 20 Universităţii Street, \\ 332006, Petrosani, \\ Romania \\ tudelena@mail.com
}

\author{
Mohammed Zaheeruddin \\ Concordia University 1455 \\ De Maisonneuve Blvd West, \\ Montreal, QC, H3G 1M8, \\ Canada \\ zaheer@encs.concordia.ca
}

\author{
Nicolae Tudoroiu \\ John Abbott College 21275 \\ Lake Shore Road, Sainte-Anne- \\ de-Bellevue, QC, H9X 3L9, \\ Canada \\ ntudoroiu@gmail.com
}

\author{
Dumitru Dan Burdescu \\ University of Craiova, 107 \\ Decebal Bvd., 200440, Craiova, \\ Romania \\ dburdescu@yahoo.com
}

\begin{abstract}
The main objective of this paper is focused on the real time implementation in a MATLAB/SIMULINK environment of a closed-loop hybrid control strategy structure consisting of a combination of Fuzzy Logic control approach and a standard PID control strategy. This strategy is applied to control the speed of a $2 \mathrm{HP} 1750 \mathrm{rpm}$ permanent magnet DC motor used in a wide range of HVAC applications. The novelty of the paper is the new modeling approach by using SIMULINK SIMSCAPE library blocks, more practical due to its simplicity, easier and faster to implement and, in particular, very easy to practice for MATLAB users compared to a traditional modelling approach. The DC motor can be powered by a unidirectional DC voltage converter connected to a $10 \mathrm{~kW}$-Microgrid PV array or directly from a Li-Ion battery or a Supercapacitor, both connected via two bidirectional DC boost-buck converters to the same Microgrid. The Microgrid energy storage system is suitable for renewable energy applications that are connected to a local grid, thus releasing the overloaded national grid, saving a considerable amount of energy and drastically reducing the energy costs.
\end{abstract}

\section{INTRODUCTION}

$\mathrm{T}$ HIS research paper investigates an alternative modelling control system design methodology for linear and nonlinear systems dynamics by using the MATLAB SIMULINK SIMSCAPE library blocks to implement a standard Proportional Integral Derivative (PID) control strategy based on a fuzzy logic control (FLC) approach. The new modelling approach is very attractive for simulations and implementations in real-time of several industrial control applications from different fields with strong interconnections to a local and national grids energy storage and communications systems. The microgrid is "widely used in generation systems, due to benefits of achieving high efficiency and increasing demand", as is stated in [1]. In fact, it is a small grid that combines a renewable resource of energy such as the most used photovoltaics (PV) to generate electrical

Research funding (discovery grant) for this project from the Natural Sciences and Engineering Research Council of Canada (NSERC) is gratefully acknowledged. power and energy storage units that are located close to the corresponding powered loads.

Preliminary results on the real time implementation of a direct current (DC) servomotor with unknown uncertainty using a sliding mode observer modelling technique and a hybrid structure of an aadaptive neuro-fuzzy modeling approach (ANFIS) applied on nonlinear dynamic systems were presented in [2] and [3]. An interesting application using a hybrid control structure as a combination of an FLC and a PID controller (FLCPID) that is connected via a DC-DC buck-boost converter to a solar power source via a battery system is developed in [4]. This application provided us with valuable information to be tailored to build the proposed Fuzzy Logic PID control strategy for a variable speed permanent DC motor (PMDCM) fan used in variable air volume (VAV) of HVAC control system applications. Fuzzy logic control-based approach is applied to improve the effective operation of the DC motor.

\section{THE PV ARRAY DC MICROGRID BRIEF DESCRIPTION}

The SIMULINK model of the integrated DC Microgrid energy storage system in a solar PV power plant it is shown in Fig.1.

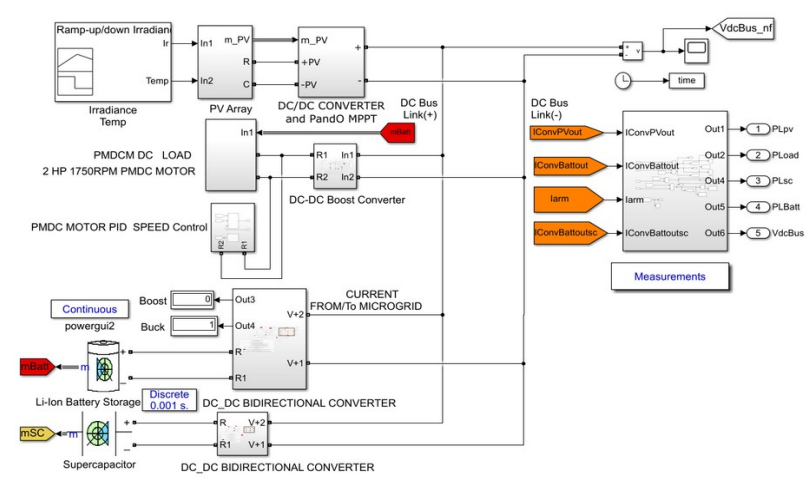

Fig.1 SIMULINK model of the integrated DC Microgrid energy storage system in a solar PV power plant 
The proposed DC Microgrid is connected to a $10-\mathrm{kW}$ PV array through an unidirectional DC-DC boost average converter, capable of generating a regulated $300 \mathrm{~V}$ DC Bus that supplies unidirectional and bidirectional DC-DC converters that control the charging and discharging of two storage power sources, Li-ion battery and Supercapacitor, and also a 2 HP 1750 rpm PMDCM - DC load.

\section{FuZZY Logic PID SpeEd CONTROL DESIGN AND MATLAB Simulations}

In this research paper the FLC is conceived as a multi-input single-output (MISO) subsystem with two inputs and one single output. The inputs of the fuzzy controller are two measured variables whose values are collected by a set of sensors integrated into a data acquisition system describing the speed error ("e") and the rate of change of the speed error ("ce"), ce $=\frac{\Delta e}{\Delta t}$, from PMDCM, the choice suggested in [5]-[6].The both inputs are then "fuzzified" using membership functions provided by an expert operator to determine the degree of membership in each input class. The resulting "fuzzy inputs" are evaluated using a linguistic rule base and fuzzy logic operations (AND, OR, NOT) to yield an appropriate fuzzy output and an associated degree of membership [7]-[8]. The "fuzzy output" of FLC is then "defuzzified" using a "centroid" gravity method to give a crisp output response to control the input signal voltage of a controlled source voltage whose output provides the regulated voltage to the PMDCM armature [8].

The fuzzy logic controller (FLC) SIMULINK model it is shown in Fig. 2.

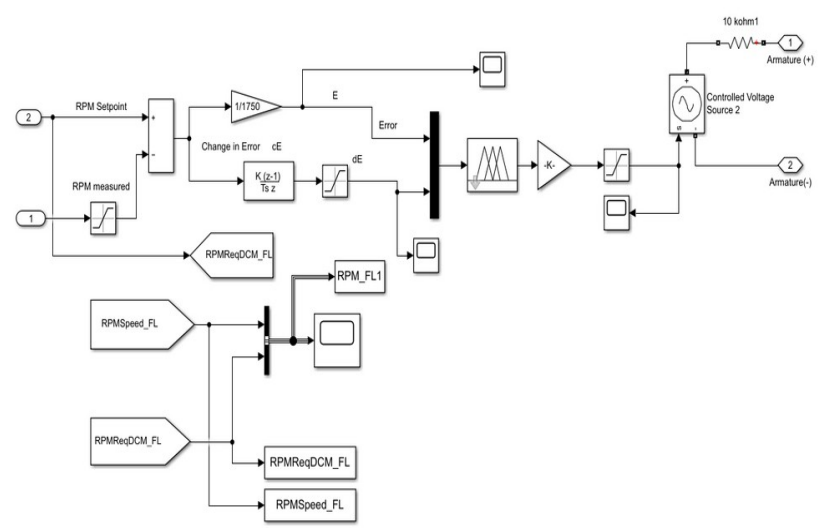

Fig.2 The Fuzzy Logic controller SIMULINK model

As it is shown in Fig.2, the inputs of the FLC, i.e. the RPM PMDCM speed error (E) and the rate of its change (cE), are converted into fuzzy linguistic variables. They are divided into a finer fuzzy partition with seven terms as it is suggested in [5]-[7], namely negative big (NB), negative medium (NM), negative small (NS), zero ( $\mathrm{ZO})$, positive small (PS), positive medium (PM) and positive big (PB). The range of the both inputs is [-1750 1750], and for output voltage is $[-300+300]$. The universe of discourse of error, rate of error and output is normalized to $[-1,1]$. The Fuzzy Logic Designer block used to implement the SIMULINK FLC model specified in Fig. 2 it is shown in Fig. 3, where the membership functions for all two inputs and the output are represented with triangle-shaped function that it is shown in Fig. 4.

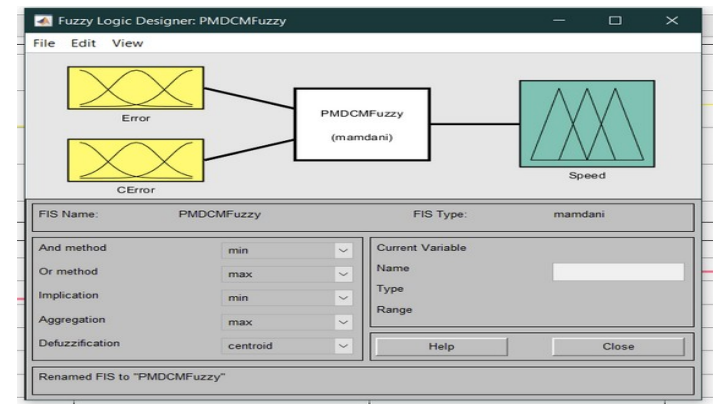

Fig.3 The Fuzzy Logic Designer block

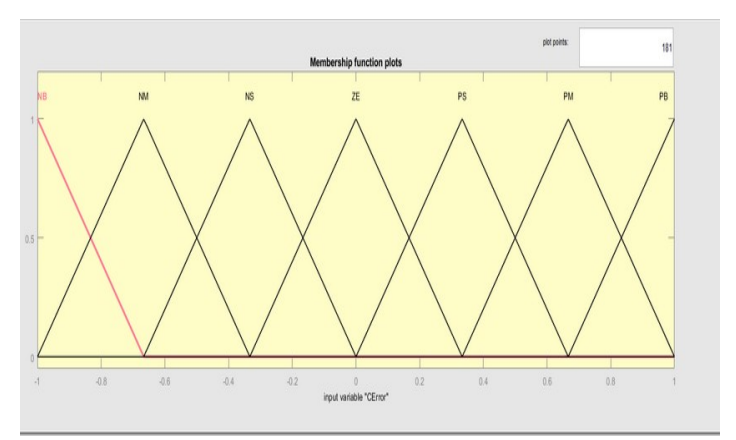

Fig. The membership function used for both inputs and the output of Fuzzy Logic controller

The optimal performance of the FLC design it is well depictured in 3-dimensional space of both FLC inputs and its output by a surface view shown in Fig. 5. The MATLAB simulation results of RPM FLC PMDCM speed control for a step response are shown in Fig. 6, and its robustness to changes in tracking setpoint and load torque it is shown in Fig.7.

In Fig. 6 it is revealed a great performance for FLC RPM speed, a very short rising and settling time, and also a very good tracking accuracy.

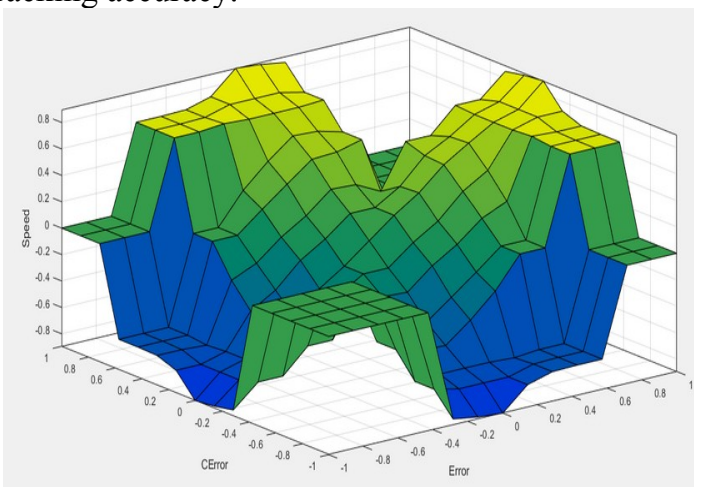

Fig. 5 The surface view of the optimal performance of the Fuzzy Logic controller 


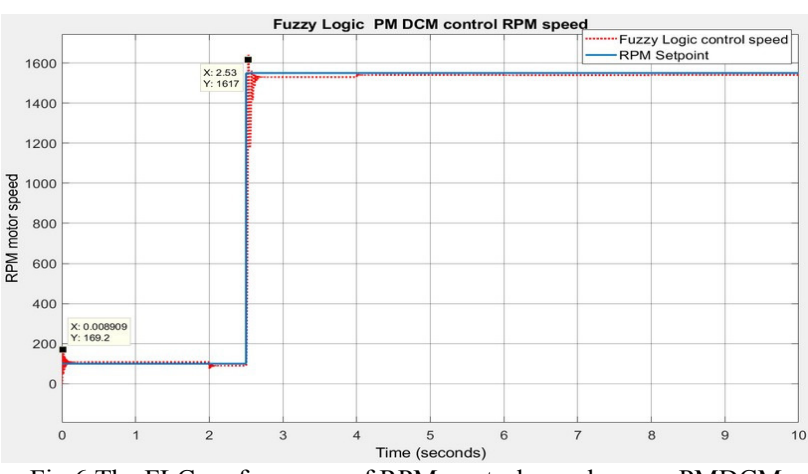

Fig.6 The FLC performance of RPM control speed versus PMDCM RPM tracking setpoint

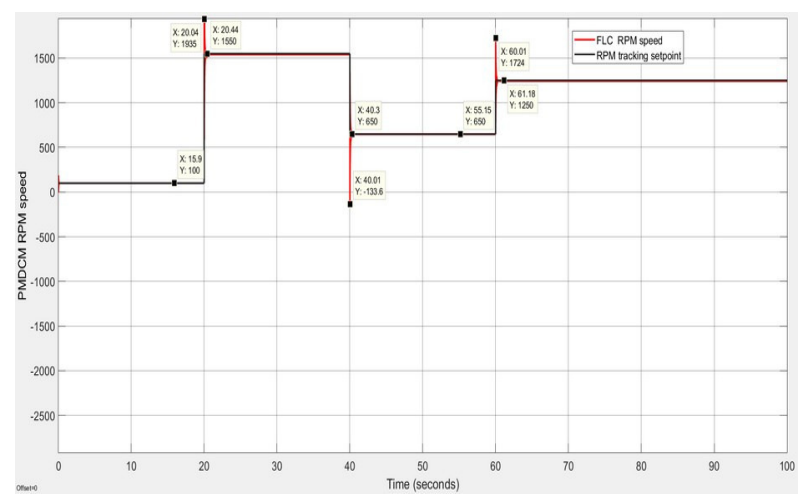

Fig.7 The FLC performance of RPM control speed versus the changes in PMDCM RPM tracking setpoint

In [9] it is suggested a combination of PID and FLC in a new structure FLPID, as it is shown in Fig.8.

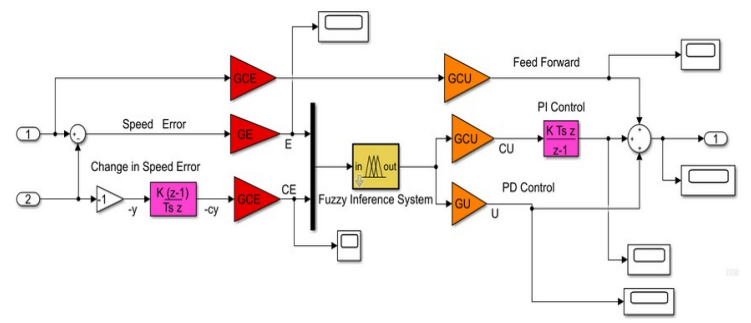

Fig.8 The Fuzzy Logic PID controller SIMULINK model (see [9])

As it is suggested in [9] the scaling factors of Fuzzy Logic PID that it is shown in Fig. 8 are calculated based on PID tuned optimal values of control parameters, $k_{p}, k_{i}, k_{d}$ using the advanced SIMULINK PID block option. Thus, the PID are set up for the following optimal values of parameters:

$k_{p}=-2.047, k_{i}=-14.7, k_{d}=-0.0487, T_{s}=0.1$ that lead

to the following values of the scaling factors [8]:

$G E=1750, G C E=G E *\left(k_{p}-\operatorname{sqrt}\left(k_{p}^{2}-4 * k_{i} * k_{d}\right)\right) / 2 / k_{i}=190.4049$

$G C U=k_{i} / G E=-0.0084, G U=k_{d} / G C E=-2.5577 \mathrm{e}-04, \mathrm{~K}=1$

The overall performance of the Fuzzy Logic PID controller it is shown in Fig. 9.

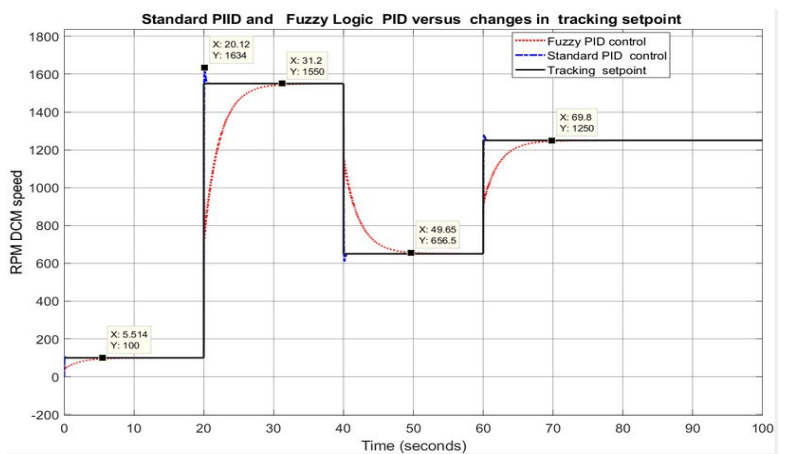

Fig.9 The Fuzzy Logic PID RPM control speed performance versus standard PID control

\section{PMDCM SPeEd CONTROL STRATEGIES PERFormance ANALYSIS}

A comparison of MATLAB simulations results for the proposed PMDCM speed (RPM) control strategies, i.e. PID, FLC and their combination FLCPID, in terms of tracking accuracy, rise and settling time responses, as well as robustness to changes in tracking reference value and load torque are shown in Fig. 10.

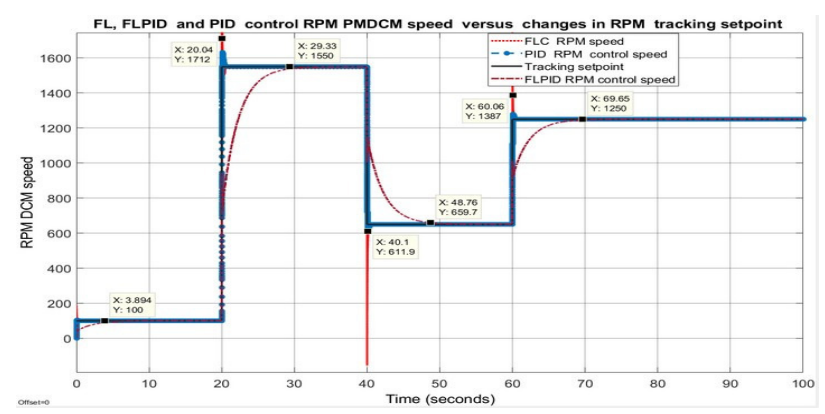

Fig.10 The PID and FLC performance of speed (RPM) control versus the changes in PMDCM RPM tracking setpoint

In the following figures Fig.11, Fig.12, and Fig. 13 there are shown the PMDCM armature current, armature voltage and the load torque profile for the combined speed (RPM) control Fuzzy Logic PID, more smooth compared to those obtained for PID and FLC separately. Also, a rigurous analysis of the performance in terms of tracking accuracy, rise time and settling time responses, and the robustness to changes in tracking setpoint and load torque indicates that PID and FLC control strategies perform slightly better compared to FLCPID, but the last one acts much smooth during the transient than first two control strategies, more useful for such of kind of applications.

\section{CONCLUSION}

In this research paper we developed a $10-\mathrm{kW}$ Microgrid of $300 \mathrm{~V}$ DC bus connected to a PV array that supplies power to a $2 \mathrm{HP} 1750 \mathrm{rpm}$ PMDCM, two storage power sources such as a Li-Ion battery and a Supercapacitor. The PV array is connected to DC Microgrid thru a DC-DC boost average 


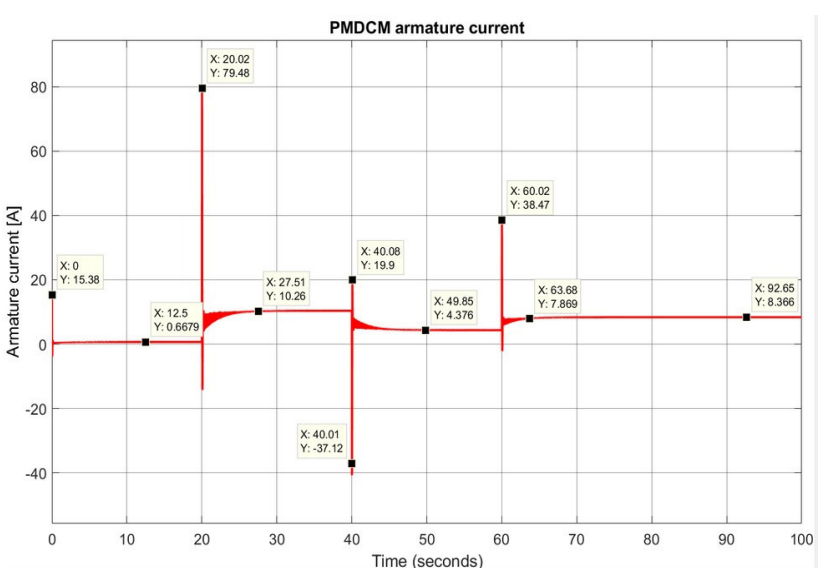

Fig.11 PMDCM armature current

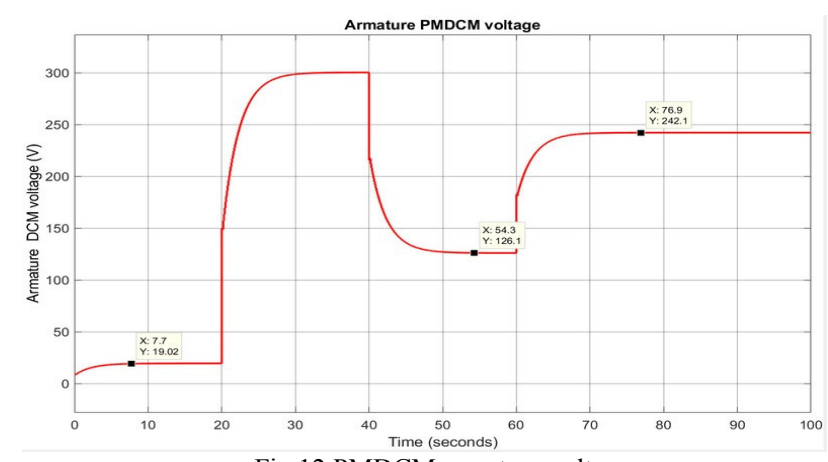

Fig.12 PMDCM armature voltage

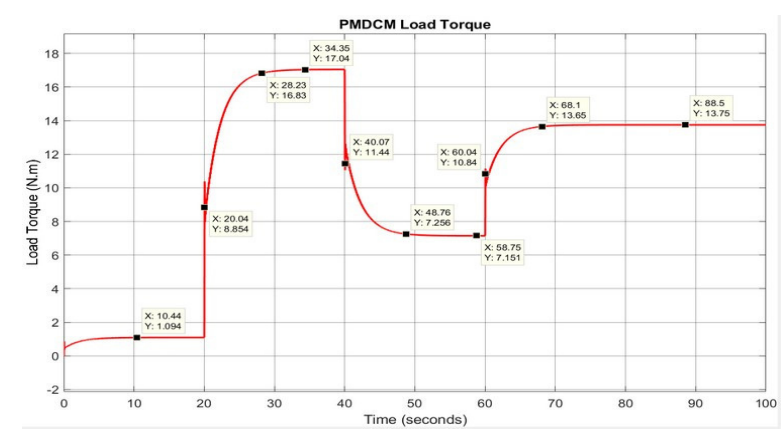

Fig.13 PMDCM load torque converter controlled by an MPPT implemented by using the simplest PandO technique. The PMDCM is connected as a DC load thru a monodirectional DC-DC boost converter, and the both storage power sources thru two bidirectional DCDC boost-buck converters. The investigation it was focused to develop for the proposed PMDCM a Fuzzy Logic speed control approach, and also a hybrid combination of the both control strategies, known as Fuzzy Logic PID controller.

The effectiveness of the proposed control strategies it was proved by intensive MATLAB simulations conducted on a MATLAB 2019a platform and SIMULINK. For future work we will be focused to extend the implementation of FLC and of the hybrid FLCPID control structure on a variable speed airflow fans used in a single zone or multi-zone VAV HVAC applications.

\section{REFERENCES}

[1] Shweta Dikshit, "Solar Photovoltaic generator with MPPT and battery Storage", International Journal of Electrical Engineering and Technology, vol.8 (3), 2017, pp. 42-49, ISSN Print: 0976-6545, ISSN online: 0976-6553.

[2] R-E Tudoroiu, W. Kecs, M. Dobritoiu, N. Ilias, S-V Casavela, N. Tudoroiu," Real-Time Implementation of DC Servomotor Actuator with Unknown Uncertainty using a Sliding Mode Observer", ACSIS, vol.8, pp.841-848, DOI: 10.15439/2016F95, Poland, 2016.

[3] E-R. Tudoroiu, M. Zaheeruddin, N. Tudoroiu, D.D. Burdescu, "MATLAB Implementation of an Adaptive Neuro-Fuzzy Modeling Approach applied on Nonlinear Dynamic Systems - a Case Study", Proceedings of the Federated Conference on Computer Science and Information Systems, pp. 577-583, September 2018, ISSN: 23005963, ACSIS, vol.15, DOI: 10.15439/2018F38.

[4] K. F. Hussein, "Hybrid Fuzzy-PID controller for Buck-Boost Converter in solar energy-Battery systems", Master's Thesis, Western Michigan University, May 2015.

[5] Huang Jiang, Wang Jie, Fang Hui, "An anti-windup self-tuning fuzzy PID controller for speed control of brushless DC motor", Automatika, 2017, vol. 58 (3), pp. 321-335, DOI: 10.1080/00051144.2018. 1423724.

[6] Chuen Chien Lee, "Fuzzy Logic in control systems: Fuzzy Logic Controller-Part 1", IEEE Transactions on Systems, Man. and Cybernetics, vol.20 (2), March/April 1990, pp. 404-418.

[7] Md. Akram Ahmad, Pankaj Rai, Anita Mahato, Megha Mahapatra, "Speed control of a DC Motor using Fuzzy Logic application", International Journal of Research in Engineering, Technology and Science, vol.7, Feb. 2017, ISSN 2454-1915, pp.1-12.

[8] Umesh Kumar Bansal, Rakesh Narvey, "Speed Control of DC Motor Using Fuzzy PID Controller", Advance in Electronic and Electric Engineering, ISSN 2231-1297, vol. 3(9), 2013, pp. 1209-1220.

[9] MathWorks Documentation, MATLAB R2019a, Fuzzy Logic Toolbox Examples/Implement Fuzzy PID Controller in Simulink Using Lookup Table - SIMULINK Library MATLAB R2019a. 